\title{
Dilation of the Cystic Duct Confluence in Laparoscopic Common Bile Duct Exploration and stone extraction for Patients with Secondary Choledocholithiasis
}

\section{Xiao-Bin Yang}

the first people's hospital,qujing

An-Shu Xu (D315000722@qq.com )

the first people's hospital,qujing https://orcid.org/0000-0002-3080-6430

Jian-Gang Li

the first people's hospital,qujing

Yong-Ping $X u$

the first people's hospital,qujing

De-Song Xu

the first people's hospital,qujing

Chao-Chun Fu

the first people's hospital,qujing

Da-Bo Deng

the first people's hospital,qujing

Jie Li

the first people's hospital,qujing

Ma-Zhong Zhang

shanghai children's medical center

Research article

Keywords: Cystic duct; Cholelithiasis; Secondary choledocholithiasis; Common bile duct exploration;

Posted Date: January 22nd, 2020

DOI: https://doi.org/10.21203/rs.2.16697/v4

License: (c) (i) This work is licensed under a Creative Commons Attribution 4.0 International License.

Read Full License 
Version of Record: A version of this preprint was published at BMC Surgery on March 17th, 2020. See the published version at https://doi.org/10.1186/s12893-020-00705-y. 


\section{Abstract}

Objectives. Many options exist in the management of cholelithiasis and secondary choledocholithiasis. Among them, laparoscopic common bile duct exploration (LCBDE) with the choledocotomy followed by laparoscopic cholecystectomy has gained popularity. However, efforts should be made for minimally invasive or non-invasive to the common bile duct (CBD). For this purpose, we modified the surgical modality of laparoscopic transcystic approach by dilating the cystic duct confluence in CBD exploration (LTD-CBDE). Based on our preliminary experience, the aim of this work was to assess the feasibility, safety and effectivity of LTD-CBDE.

Patients and methods. Sixty-eight patients were arbitrarily offered new LTD-CBDE technique from December 2015 to April 2018. During the surgery, we dilated the cystic duct confluence with separation forceps and/or the columnar dilation balloon. Subsequently, the CBD exploration and stone extraction were performed with choledochoscope. The entrance of CBD was covered with cystic duct stump wall and primarily closed at the end of surgery.

Results. 49 females and 19 males with cholelithiasis and secondary choledocholithiasis were included. The mean age was 53 years old (18 to $72 \mathrm{yr}$ ). Of which, 62 cases $(91.2 \%)$ were performed with LTD-CBDE techniques successfully, bile leakage was observed in 3 cases (4.4\%). The mean operation time was 106 minutes and the mean hospital stay was 5.9 days. As for the other 6 patients, 3 cases were converted to open cholecystectomy due to severe fibrosis, unclear anatomical structure at the Calot's triangle $(n=2)$ and Mirizze syndrome $(n=1)$; LCBDE were performed in 3 patients due to cystic duct atresia $(n=2)$ and low level of the gallbladder duct into CBD $(n=1)$. These patients had smooth postoperative course. None of the patients presented radiological evidence of retained CBD stone on the postoperative follow-up in 43/68 (40 cases used LTD-CBDE) patients one year later.

Conclusion: The current work suggests that LTD-CBDE for the management of cholelithiasis and secondary choledocholithiasis is a feasible, safe and effective technique with a low rate of complications. It offers another alternative for surgeons to treat patients in similar scenarios. However, additional randomized, controlled study is further needed to demonstrate its efficacy, safety, and impact on CBD stenosis.

\section{Introduction}

The incidence of common bile duct (CBD) stones reported in the literature in patients with gall bladder stones varies between $7 \%$ and $20 \%{ }^{[1-3]}$. Shortly after Bobbs performed the first cholecystectomy in 1867 , Abbe performed the first CBD exploration ${ }^{[4]}$. Subsequently, open cholecystectomy and CBD exploration followed by T-tube drainage gradually became a classic surgical modality for patients with cholelithiasis and secondary choledocholithiasis. With the introduction of endoscopy and laparoscopy into clinic in the 1970 s and 1980s, as also accompanied by the evolution of widespread expertise, laparoscopic cholecystectomy (LC) has been rapidly accepted as a routine treatment for patients with symptomatic 
gall bladder stones, and has also gradually as the conventional management of CBD stone ${ }^{[5]}$. In China, Yunnan Province is a high-prevalence area of lithiasis. A hospital in Qujing city introduced laparoscopic techniques to China first in 1991, and more than 100 cases of LC were performed that year.

Several clinical management methods are available for patients with cholelithiasis and secondary choledocholithiasis. Although the ideal method is still in dispute, endoscopic retrograde cholangiopancreatography (ERCP, two-stage) and laparoscopic common bile duct exploration (LCBDE) with the choledocotomy followed by LC (LCBDE, single-stage) are two commonly used methods [5-11]. Compared with ERCP followed by LC, LCBDE has the advantage of a higher success rate and a shorter hospital stay while simplifying the two procedures into a single minimally invasive operation ${ }^{[5]}$. However, in most cases, the LCBDE modality usually has to be performed via a choledochotomy followed by T-tube drainage. T-tube placement presents difficulties in post-operative management ${ }^{[12]}$. Thus, Chen et al. ${ }^{[13]}$ and Niu et al. ${ }^{[14]}$ modified transcystic approach with micro-incision of the cystic duct confluence or CBD followed by primary suture without a T-tube. Results had achieved good preliminary effects. Despite this, we think that micro-incision is still a kind of injury to $C B D$, and in the long run, patients might suffer from CBD stenosis, especially for those patients with CBD stenosis before operation.

Therefore, efforts should be made to minimize the injury to the cystic duct confluence or CBD, preferably "no injury". For this purpose, we modified the surgical modality by replacing choledochotomy or confluence incision with Laparoscopic Transcystic Dilation of the cystic duct confluence in CBDExploration (LTD-CBDE). The basic idea is that since secondary CBD stone originates from gall bladder stone and in some cases the stones will fall through the cystic duct into the CBD, indicating that if the natural cystic duct and the cystic duct confluence can be safely dilated, possibly the stones will be found and removed through choledochoscope. The improved procedure takes full advantage of the natural tube of the cystic duct and avoids incision CBD. In this article, we report our preliminary experience during the last three years, and assess the feasibility, safety and effectivity of LTD-CBDE technique.

\section{Patients And Methods}

This study was approved by the Institutional Review Board of The Qujing First People's Hospital, and was considered not requiring informed consent given the retrospective nature of using patients' data. From Dec. 2015 and Apr. 2018, 191 patients in total were diagnosed as cholelithiasis and secondary choledocholithiasis by preoperative ultrasound (US) and/or magnetic resonance cholangiopancreatography (MRCP) examinations and refused ERCP. In 9 cases CBD stones were successfully extracted through cystic duct and confluence with choledochoscope, while 68 cases were arbitrarily offered LTD-CBDE, and the other 114 cases still underwent classic single-stage LCBDE. Meanwhile, the outer diameter, location and size of the CBD stones were also clearly defined and recorded. The patients presented with acute cholangitis and acute gallbladder pancreatitis were excluded from undergoing LTD-CBDE. All patients underwent routine preoperative examination, including chest X- 
ray, electrocardiogram, routine blood test, liver and kidney function testing, and coagulation function testing. All patients were informed about both the procedures and the technologies possibly being selected and provided written informed consent.

Operations were performed by the same surgical team under general anesthesia and endotracheal intubation. During the operation, the patients were placed in reverse trendelenburg positions, tilting to the left. Pneumoperitoneum was established with carbon dioxide at a pressure of $12-15 \mathrm{mmHg}$ and adjusted as needed. Four trocars were used for LC according to the standard technique. The detailed surgery flow diagram is shown in figure 1 . The Calot's triangle was dissected, and the cystic artery, cystic duct and CBD were exposed. The cystic artery was clipped and ligated first. Then, the cystic duct was clipped highly near the GB, and $3 / 4$ of its cross section was cut open approximately $0.5 \mathrm{~cm}$ from the CBD. The choledochoscope (4.9 mm, Olympus, Tokyo, Japan) was inserted through the cystic duct and confluence for the CBD exploration and stone extraction (success in 9 cases). Stones were removed with a Cook basket (NTSE-045065-UDH). If failed, one of next steps were performed: (i) LCBDE modality $(n=114)$; or (ii) the ventral side of the cystic duct was longitudinally incised $0.2-0.3 \mathrm{~cm}$ from the above-mentioned incision of cross section, and the confluence was dilated through cystic duct with the separation forceps (figure 2), and then the choledochoscope was inserted again; or (iii) columnar dilation balloon (M00558410) was used to dilate the confluence (figure 3) until the choledochoscope was successfully inserted followed by the CBD exploration and stone extraction. The choledochoscope was also turned upward to explore the common hepatic duct and intrahepatic ducts. If intrahepatic ducts present no stones, then the distal CBD was explored again. Intraoperatively, the 68 patients reported herein (red dotted box in figure 1) were selected arbitrarily to adopt the surgical methods (LTD-CBDE modality) described in (ii) and (iii) above. As far as our practical experience was concerned, the dilation degree of the cystic duct and/or the confluence depended on the size of the largest stone individually, which was determined basing on preoperative US or MRCP examination. After the stones were removed totally (the CBD was completely clear and no evidence of remnant stones), the cystic duct was cut and LC was performed. The stump wall of the cystic duct was used to cover the entrance of CBD, primarily by laparoscopic interrupted full-thickness suturing, as shown in figure 4. The abdominal drainage tube was left at the small omentum hole in all patients. Those interested can refer to the full video shown in supplemented material.

The patients' demographic data and the following indicators were collected and recorded: operation time in minutes, surgical success rate, open conversion rate, residual stone rate, timing of postoperative return of peristaltic activity and food intake, postoperative hospital stay, and postoperative bile leakage rate. Before the patients were discharged from the hospital, they were instructed to pay attention to the clinical symptoms, signs, laboratory and imaging findings related to possible biliary diseases after surgeries. A follow-up MRCP evaluation was done a year after surgery. Data are presented with numbers, percentage, arithmetic mean \pm standard deviation (SD).

\section{Results}


Sixty-eight patients with cholelithiasis and secondary choledocholithiasis were arbitrarily offered LTDCBDE. Table 1 shows patient demographics and characteristics of lithiasis. The patient population comprised $19(27.9 \%)$ males and $49(73.1 \%)$ females, with mean age $53 \pm 14$ years old (ranged between 18 and $72 \mathrm{yr}$ ). Among them, 52 patients presented with symptoms and signs of obstructive jaundice, and 8 patients had previous abdominal surgery history. The gall bladder stone was confirmed by US examinations, and the CBD stone was initially diagnosed by US and confirmed again by MRCP in all patients. The mean measured outer diameter of CBD was $12.6 \pm 1.8 \mathrm{~mm}$ (ranged from 9 to $17 \mathrm{~mm}$ ). The median largest diameter of CBD stone was $9 \mathrm{~mm}$ (ranged from 0.3 to $1.1 \mathrm{~cm}$ ).

Of the 68 patients, 62 cases (91.2\%) were performed LTD-CBDE successfully, and the CBD dilated with columnar dilation balloons (in 5 patients) and separation forceps (in 57 patients), respectively. The mean operation time was 106 minutes (ranged from 90 to 120 minutes). Patients with ASA III grade would stay in the ICU for 1-4 days and the rest would return to the ward. All patients regained peristaltic activity in the next postoperative day, and took liquid food next early morning. The mean postoperative hospital stay was 5.9 days (4-15 days). The outcomes related to modified LTD-CBDE modality are shown in table 2 .

As shown in table 3, among the 62 patients who successfully performed LTD-CBDE, bile leakage was observed in 3 cases (4.4\%). The maximum bile drainage volume in these 3 patients was less than 100 $\mathrm{mL} /$ day and the bile leakage stopped within 2 days. The abdominal drains were removed within $24-48 \mathrm{~h}$ postoperatively. Of all 62 patients had no evidence of residual stones and CBD stenosis with routine MRCP examinations before discharge. 40 cases returned to the hospital for MRCP re-examination one year after surgery, and none showed signs of CBD recurrence or stenosis.

Of 68 patients included, 6 patients (8.8\%) were treated by other surgical methods for various reasons. In 2 cases with fibrosis and unclear anatomical structures at the Calot's triangle and in 1 case with coexisting Mirizze syndrome, it's converted to open cholecystectomy and CBD were explored to remove the stones safely with T-tube drainage; In 2 cases with cystic duct atresia and in 1 case with low confluence opening, LCBDE and T-tube drainage were performed. Postoperative MRCP revealed patency of the CBD. These six patients had smooth postoperative course. 3 cases returned to the hospital for MRCP re-examination one year after surgery, and none showed signs of CBD recurrence or stenosis.

\section{Discussion}

The secondary CBD stone may cause many clinical symptoms and signs, including abdominal pain, obstructive jaundice, cholangitis, and biliary pancreatitis, etc ${ }^{[2]}$. Like patients in this article, there were as many as 52 patients diagnosed as obstructive jaundice. The ideal management remains a matter of debate ${ }^{[5]}$. As far as LCBDE is concerned, the problems are mainly related to T-tube placement, such as patient discomfort, biliary peritonitis, T-tube displacement, etc ${ }^{[15-16]}$. In view of this, some surgeons tried to make full use of the natural cystic duct with micro-incision on CBD or confluence followed by primarily suture ${ }^{[13-14]}$. Inspired by this, we further speculate: could we use the natural orifices comprising of cystic duct and its confluence at the CBD with no incision? Therefore, LTD-CBDE was designed for CBD 
exploration and stones extraction via laparoscopic transcystic approach by dilating the confluence. Dilation of the confluence making the insertion of the choledochoscope and stones extraction easier, because is it not only overcomes the problems that the cystic duct is thin and the spiral valve acts as a barrier in exploration, but also enlarges the inlet diameter of the CBD greater or equal to the outer diameter of the largest stone. CBD blood supply is not affected by incision so that CBD stenosis may be prevented. The operations were performed smoothly. Success rate was $91.2 \%$ (may be higher with careful perioperative identification of the indications), clearance rate of CBDS was $100 \%$, and retained stones were not identified on postoperative follow-up.

The success rate of $91.2 \%$ with present method coincides with $88.1 \%$ reported in meta-analysis of eleven randomized trials for $\mathrm{LCBDE}^{[5]}$. By using a slightly different approach with a micro-incision at the confluence, Chen et al. and Niu et al reported a success rate of $100 \%{ }^{[13-14]}$. The 6 failed patients in our work were associated with anatomical problems, suggesting the importance of a carefully selected surgical strategy. Special attention should be paid to the following aspects: Firstly, it is crucial to maintain the cystic duct intact, so as to facilitate the incision, dilation, choledochoscope insertion, observation, stones extraction and primary closure. Secondly, identify the confluence correctly and avoid its damage. Finally, limiting factors of success with LTD-CBDE include anatomic features related to the cystic duct and confluence, such as fibrosis and anatomical abnormality of the Calot's triangle, small-size or atretic or tortuous duct, and low level of or posterior insertion of the cystic duct on the CBD, etc.

The operation time reported in the literatures varies widely depending on the surgical method, ranging from 104 to 194 minutes $[2,13,17,18]$. The mean operating time was 105 minutes in our series. However, we don't think it is reasonable to directly compare the operation time, because any new modality requires more operation time, and is technically difficult with a clear learning curve. In the future, along with technical improvement and more effective logistic organization, the operation time will be further reduced. Patients in our study were discharged after a mean postoperative hospital stay of 5.9 days, which is not longer than other reports with mini-incision (mean 8 days) or $\operatorname{LCBDE}[5,17,18]$. Mortality is also in accordance with the findings of other surgical modality ${ }^{[13-14]}$. Forty-three cases were followed up one year after LTD-CBDE operation (25 patients lost to follow up) and none of them presented with evidence of retained or recurrent CBDS and stenosis of $\mathrm{CBD}$.

Our LTD-CBDE is safe and effective, but a carefully selected surgical strategy should be especially emphasized as suggested by Gigot et al. ${ }^{[18]}$. Firstly, for patients with anatomical abnormalities or intraperitoneal adhesions as 6 patients shown in our series, the traditional open operation or laparoscopic choledochotomy should be considered as soon as possible. Secondly, despite careful suturing of the confluence with stump wall, there were still 3 cases suffering postoperative bile leakage. This is not higher than the incidence of LCBDE (5.6\% with experienced surgeons vs $17.1 \%$ with inexperienced surgeons) reported by liu et al. ${ }^{[19]}$. As analyzed by liu. et al. ${ }^{[19]}$, it is clear that postoperative bile leakage (and the like) can be reduced with gaining of experience in the technique ${ }^{[20]}$. Thirdly, in our series, the separation forceps, rather than the balloons, were used in most patients. It is undeniable that the latter 
provides a quantitative and accurate degree of expansion, thus improving safety accordingly. However, Yunnan is a poor province in China, and most patients cannot afford the expensive balloon. Therefore, we

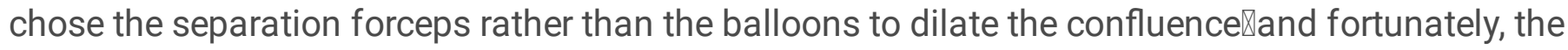
majority of patients, who used the separation forceps to dilate the confluence have achieved successfully surgery with LTD-CBDE technique. Fourthly, this is a retrospective analysis. The surgical method was selected subjectively rather than randomly, and that's why we did not take the remaining 114 patients with LCBDE as a control. Robust RCT research will be our next goal. Finally, although we hypothesized that LTD-CBDE has the potential to reduce postoperative bile duct stricture, this is currently not confirmed and further research is needed. The optimal management of CBDS depends on the skills and techniques of the surgical team available. In any case, minimally invasive or non-invasive procedures should be the direction of our efforts.

\section{Conclusions}

We modified the surgical modality by dilating the cystic duct confluence in CBD exploration (LTD-CBDE). It is a safe and effective surgical procedure for patients with secondary choledocholithiasis, although it requires clinical experience as well as advanced laparoscopic skills. However, additional randomized, controlled study is needed to demonstrate its efficacy, safety, and impact on CBD stenosis, especially in patients with preoperative CBD stricture.

\section{Declarations}

\section{Acknowledgments}

We would like to thank Professor Nin Ma (Department of Anesthesiology, Shanghai Children's Medical center, Shanghai Jiao Tong University School of Medicine) for his guidance and advice.

\section{Authors' contributions}

Yang XB, Xu AS, Li JG, Xu YP, Xu DS, Fu CC, Deng DB, Li J, and Zhang MZ contributed to the concept and design of the study. Yang XB, Xu AS, Xu YP and Fu CC contributed to clinical information. All authors contributed to the writing of the manuscript. All authors read and approved the final manuscript.

\section{Funding}

No funding.

\section{Availability of data and materials}

The data that support the findings of this study are available from Department of General Surgery, The First People's Hospital, Qujing City, Yunnan Province, China, however, restrictions apply to the availability of this data; which was used under license for the current study, therefore are not publicly available. Data 
are however available from the authors upon reasonable request, with permission from the Department of General Surgery.

\section{Ethics approval and consent to participate}

This study was approved by the Institutional Review Board of The Qujing First People's Hospital\and was considered not requiring informed consent for the retrospective nature of using patients' data.

\section{Consent for publication}

Written informed consent for publication was obtained from all participants.

\section{Competing interests}

The authors declare no conflicts of interest.

\section{Author details}

1 Department of General Surgery, The First People's Hospital, Qujing City, Yunnan Province, China 65500. 2 Department of Anesthesiology, The First People's Hospital, Qujing City, Yunnan Province, China 655000.

\section{Abbreviations}

LTD-CBDE: laparoscopic transcystic approach by dilating the cystic duct confluence in CBD exploration; LCBDE: laparoscopic common bile duct exploration with the choledocotomy followed by laparoscopic cholecystectomy; CBD: common bile duct囚LC: laparoscopic cholecystectomy; US: ultrasound; ERCP: endoscopic retrograde cholangiopancreatography; MRCP: magnetic resonance cholangiopancreatography.

\section{References}

1. Neuhaus H, Feussner $H$, Ungeheuer A, Hoffmann W, Siewert JR, Classen M. Prospective evaluation of the use of endoscopic retrograde cholangiography prior to laparoscopic cholecystectomy. Endoscopy 1992; 24(9): 745-749

2. Ghazal AH, Sorour MA, El-Riwini M, El-Bahrawy H. Single-step treatment of gall bladder and bile duct stones: A combined endoscopic-laparoscopic technique. Int J Surg 2009; 7(4): 338-346

3. Sarli L, Pietra N, Franze A, Colla G, Costi R, Gobbi S, Trivelli M. Routine intravenous cholangiography, selective endoscopic retrograde cholangiography and endoscopic treatment of common bile duct stones before laparoscopic cholecystectomy. Gastrointest Endosc 1999; 50: 200-208

4. Ellis PH. The story of gallstones and their treatment. J Perioper Pract 2019 (Epub ahead of print)

5. Singh AN, Kilambi R. Single-stage laparoscopic common bile duct exploration and cholecystectomy versus two-stage endoscopic stone extraction followed by laparoscopic cholecystectomy for patients 
with gallbladder stones with common bile duct stones: systematic review and meta-analysis of randomized trials with trial sequential analysis. Surg Endosc 2018; 32(9): 3763-3776

6. Sevinc B, Karahanö. The role safety steps in success and complication rate of endoscopic retrograde cholangiopancreatography. Surg Aparosc Endosc Percutan Tech 2017; 27(4): 233-236

7. Guda NM, Reddy DN, Kumar A. Complications of ERCP. Indian J Gastroenterol 2014; 33(1): 1-9

8. Andriulli A, Leandro G, Niro G, Mangia A, Festa V, Gambassi G, Villani MR, Facciorusso D, Conoscitore P, Spirito F, De Maio G. Pharmacologic treatment can prevent pancreatic injury after ERCP: a metaanalysis. Gastrointest Endosc 2000; 51: 1-7

9. Dickinson RJ, Davies S. Post-ERCP pancreatitis and hyperamylasaemia: the role of operative and patient factors. Eur J Gastroenterol Hepatol 1998; 10: 423-8

10. Sherman S, Hawes RH, Rathgaber SW, Uzer MF, Smith MT, Khusro QE, Silverman WB, Earle DT, Lehman GA. Post-ERCP pancreatitis: randomized, prospective study comparing a low- and highosmolality contrast agent. Gastrointest Endosc 1994; 40: 422-7

11. Nzenza TC, Al-Habbal Y, Guerra GR, Manolas S, McQuillan T. Recurrent common bile duct stones as a late complication of endoscopic sphincterotomy. BMC Gastroenteroloy 2018; 18: 39

12. Podda M, Polignano FM, Luhmann A, Wilson MS, Kulli C, Tait IS. Systematic review with metaanalysis of studies comparing primary duct closure and T-tube drainage after laparoscopic common bile duct exploration for choledocholithiasis. Surg Endosc 2016; 30(3): 845-861

13. Chen XM, Zhang Y, Cai HH, Sun DL, Liu SY, Duan YF, Yang C, Jiang Y, Wu HR. Transcystic approach with micro-incision of the cystic duct and its confluence part in laparoscopic common bile duct exploration. J Laparoendosc Adv Surg Tech A 2013; 23: 977-981

14. Niu X, Song J, He X, Chen J, Xu J, Li Z, Long H, Wei J. Micro-incision of the cystic duct confluence in laparoscopic common bile duct exploration for elderly patients with choledocholithiasis. Indian J Surg 2018; 80(3): 227-232

15. Decker G, Borie F, Millat B, et al. One hundred laparoscopic choledochotomies with primary closure of the common bile duct. Surg Endosc 2003; 17: 12-18

16. Hiromi T, Akiko U, Hui C, et al. Laparoscopic management of common bile duct stones: transcystic approach and choledochotomy. J Hepatobiliary Pancreat Surg 2002; 9: 206-212

17. Darkahi B, Liljeholm H, Sandblom G. Laparoscopic common bile duct exploration: 9 year experience from a single center. Front Surg 2016; 3: 23

18. Gigot JF. Navez B, Etienne J, Cambier E, Jadoul P, Guiot P, Kestens PJ. A stratified intraoperative surgical strategy is mandatory during laparoscopic common bile duct exploration for common bile duct stones. Lessons and limits from an initial experience of 92 patients. Surg Endosc 1997; 11(7): $722-728$

19. Liu D, Cao F, Liu J, Xu D, Wang Y, Li F. Risk factors for bile leakage after primary closure following laparoscopic common bile duct exploration: a retrospective cohort study. BMC Surg 2017; 17(1): 1 
20. Zhu H, Wu L, Yuan R, Wang Y, Liao W, Lei J, Shao J. Learning curve for performing choledochotomy bile duct exploration with primary closure after laparoscopic cholecystectomy. Surg Endosc 2018; 32(10): 4263-4270.

\section{Tables}

Table 1 Patient demographics and characteristics of lithiasis $(\mathrm{n}=68)$

\begin{tabular}{ll}
\hline Variable & Value \\
\hline Gender & $19(27.9 \%)$ \\
Men & $49(72.1 \%)$ \\
Women & $53 \pm 14(18-72)$ \\
Mean age, year (range) & $8(11.8 \%)$ \\
Previous abdominal surgery history & $42 / 20 / 6$ \\
ASA I/II/III & \\
Number of CBD & 54 \\
1 & 12 \\
$2-3$ & 2 \\
$>3$ & $9(3-11)$ \\
Median diameter of largest stone, mm (range) & 9 \\
\hline
\end{tabular}

Values are expressed as mean $\pm \mathrm{SD}$, median or number of patients (\%); ASA: American Society of Anesthesiologists physical status; CBD: common bile duct.

Table 2 The outcomes related to modified surgical modality $(\mathrm{n}=62)$

\begin{tabular}{ll}
\hline Variable & Value \\
\hline Mean operation time, minutes (range) & $106 \pm 9(90-120)$ \\
Success rate & $62 / 68(91.2 \%)$ \\
Common bile stone clearance rate & $62(100 \%)$ \\
Retained stones with postoperative MRCP & $0(0 \%)$ \\
Mean postoperative hospital stay, day (range) & $5.9 \pm 2.4(4-15)$ \\
\hline
\end{tabular}

Values are expressed as mean \pm SD or number of patients (\%); MRCP: magnetic resonance cholangiopancreatography.

Table 3 The complications related to modified surgical modality and treatments of failure cases

\begin{tabular}{ll}
\hline Variable & Value \\
\hline Complications & $0 /(0 \%)$ \\
Pancreatitis & $3 / 62(4.8 \%)$ \\
Biliary leakage & $3 / 68(4.4 \%)$ \\
Conversion open rate & $3 / 68(4.4 \%)$ \\
Conversion Choledochotomy + T-tube & $43 / 68(63.2 \%)$ \\
\hline Number of follow-up one year later & 4
\end{tabular}

Values are expressed as number of patients (\%). 


\section{Figures}

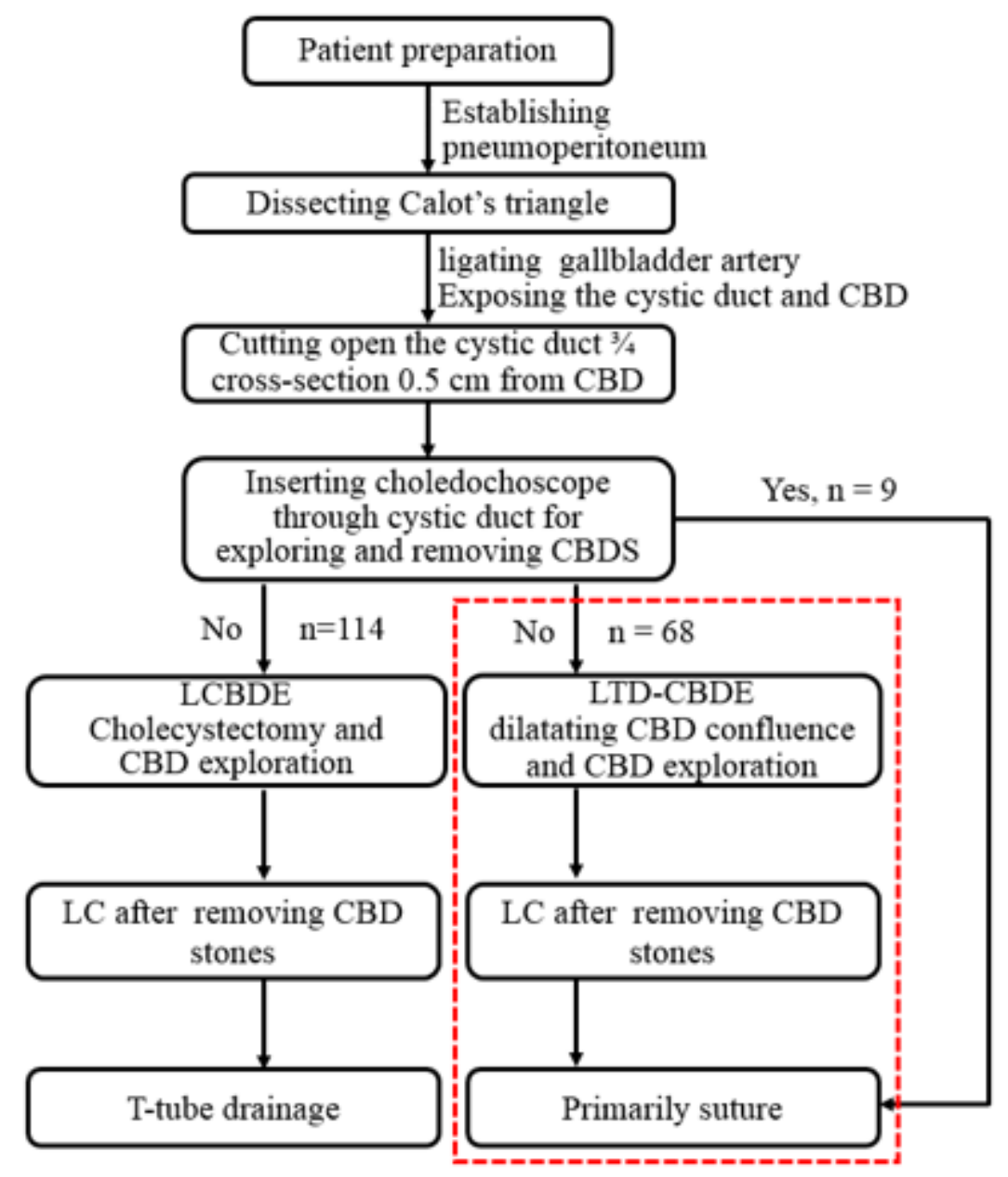

\section{Figure 1}

The detailed surgery flow diagram. Among 191 patients with cholelithiasis and secondary choledocholithiasis, the CBD stones were successfully extracted through cystic duct and confluence with choledochoscope in 9 cases, while 68 cases received LTD-CBDE (red dotted box) and 114 cases received $\angle C B D E$, respectively. CBD, common bile duct; $\angle C B D E$ : laparoscopic $C B D$ exploration with the choledocotomy followed by laparoscopic cholecystectomy; LTD-CBDE: laparoscopic transcystic approach by dilating the cystic duct confluence in CBD exploration. LC: laparoscopic cholecystectomy. 


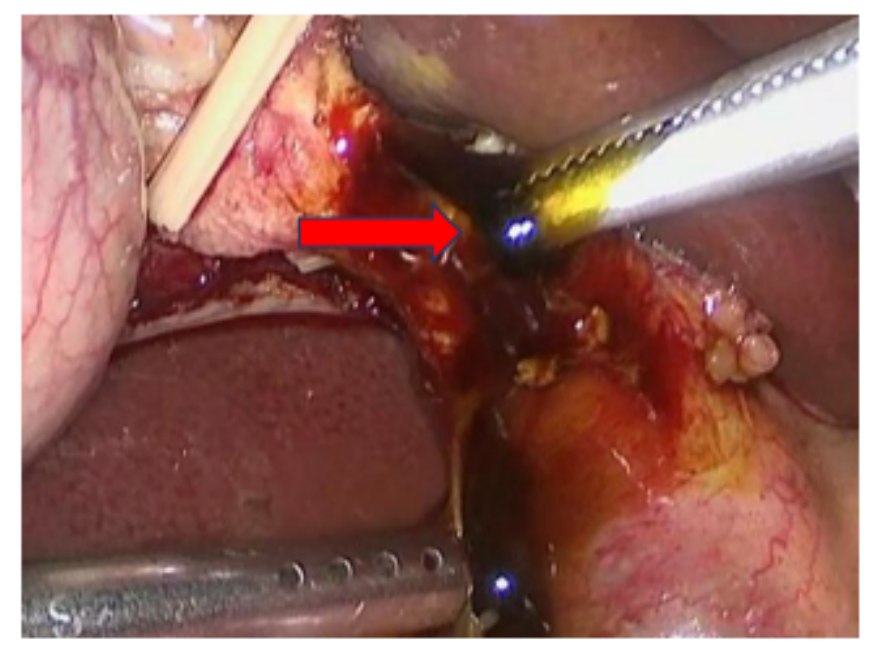

Figure 2

The confluence was dilated with the separation forceps (red arrow)

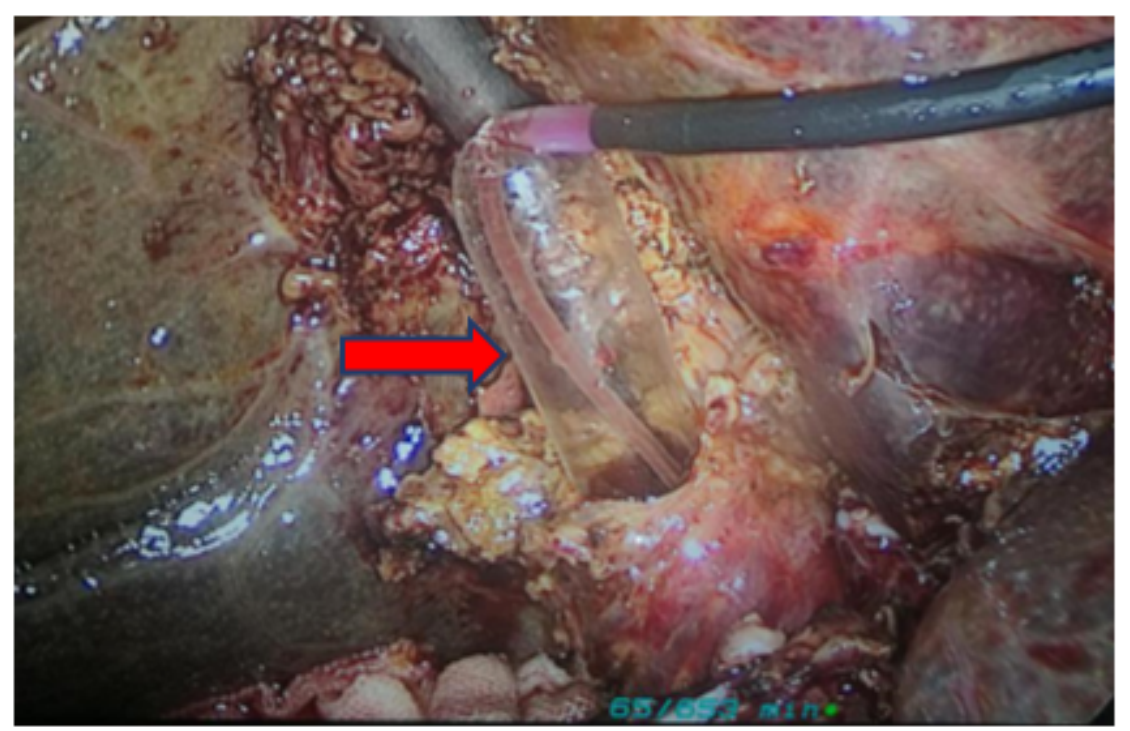

Figure 3

The confluence was dilated with the columnar dilation balloon (red arrow) 


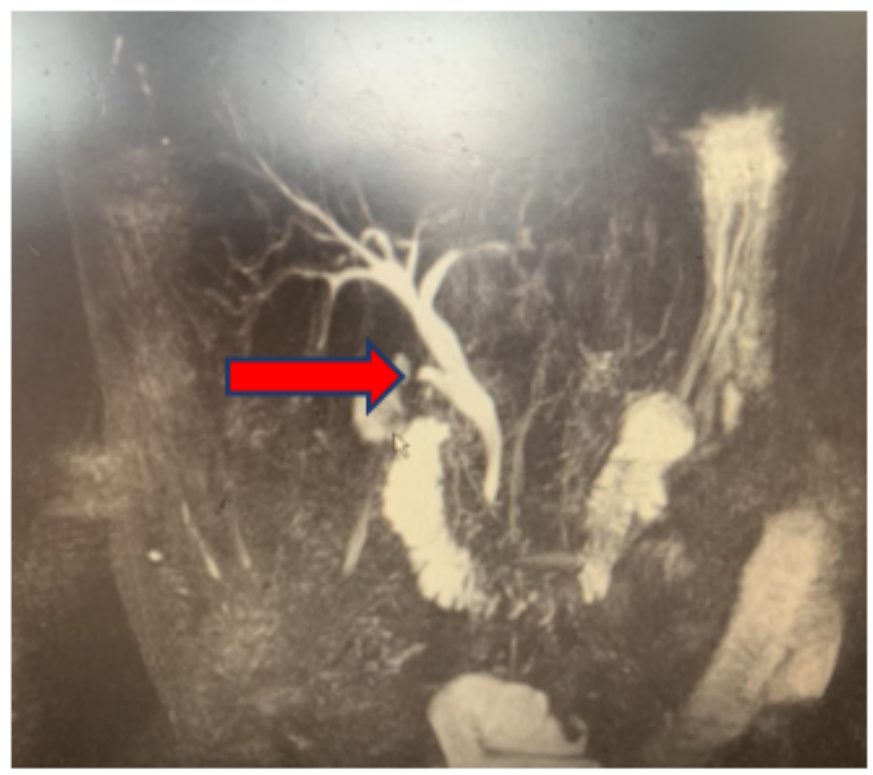

\section{Figure 4}

The stump wall of the cystic duct covers the entrance of CBD followed by primarily close. Postoperative magnetic resonance cholangiopancreatography (MRCP) examination shows good healing (red arrow).

\section{Supplementary Files}

This is a list of supplementary files associated with this preprint. Click to download.

- video.mp4 